\title{
Low maximal oxygen uptake is associated with elevated depressive symptoms in middle-aged men
}

\author{
T. Tolmunen ${ }^{1}$, J. A. Laukkanen ${ }^{2}$, J. Hintikka ${ }^{1,3,4}$, S. Kurl ${ }^{2}$, H. Viinamäki ${ }^{1}$, R. Salonen ${ }^{2}$, \\ J. Kauhanen ${ }^{2}$, G. A. Kaplan ${ }^{5}$ and J. T. Salonen ${ }^{2,6}$ \\ ${ }^{1}$ Department of Psychiatry, Kuopio University Hospital, P.O. Box 1777, FIN-70211, Kuopio, Finland; ${ }^{2}$ Research Institute of \\ Public Health, Department of Public Health and General Practice, University of Kuopio, Kuopio, Finland; ${ }^{3}$ Department of \\ Psychiatry, University of Tampere, Finland; ${ }^{4}$ Department of Psychiatry, Paijat-Hame Central Hospital, Lahti, Finland; \\ ${ }^{5}$ Department of Epidemiology, Institute for Social Research Center for Social Epidemiology and Population Health, University \\ of Michigan School of Public Health, US; ${ }^{6}$ Inner Savo Health Centre, Suonenjoki, Finland
}

Accepted in revised form 5 July 2006

\begin{abstract}
A low level of physical activity has been associated with depression, and increased physical activity has been found to have a positive effect on mood. However, the association between maximal oxygen uptake $\left(\mathrm{VO}_{2 \max }\right)$ and mood has been poorly studied. In this study $\mathrm{VO}_{2 \max }(\mathrm{ml} / \mathrm{kg}$ per min) was measured in a sample of 1,519 men aged 46-61 years during a cycle ergometer test by using respiratory gas exchange. Men with a history of psychiatric disorder or serious physical illness were excluded. Depressive symptoms were assessed using the 18-item Human Population Laboratory Depression Scale (HPL).
\end{abstract}

Those who scored 5 or more in the HPL were considered to have elevated depressive symptoms.

The participants were classified into quartiles according to the $\mathrm{VO}_{2 \max }$. Those in the lowest quartile had a more than 3-fold (OR: 3.42; 95\% CI: 1.65-7.09; $p<0.001)$ higher risk of having elevated depressive symptoms compared with those in the highest quartile, even after adjusting for several confounders (OR: 3.38; 95\% CI: $1.60-7.14 ; p<0.001)$.

In conclusion, low $\mathrm{VO}_{2 \max }$ is associated with having elevated depressive symptoms in middle-aged men.

Key words: Depression, Cardiovascular fitness, Maximal oxygen uptake, Physical activity

Abbreviations ANOVA = analysis of variance; BMI = body mass index; $\mathrm{CI}=$ confidence interval; $\mathrm{HPL}=$ Human Population Laboratory; KIHD = The Kuopio Ischaemic Heart Disease Factor; MET = metabolic equivalent; $\mathrm{OR}=$ odds ratio; $\mathrm{SES}=$ sosioeconomic status; $\mathrm{VO}_{2 \max }=$ maximal oxygen uptake

\section{Introduction}

In population studies, regular physical exercise has been related to a lower level of depressive symptoms [1-4]. It has been suggested that this association might be especially strong among older subjects [5-7]. Physical exercise has also been applied experimentally as a treatment for depression, either alone $[8,9]$ or as an augmentation of anti-depressive medication or psychotherapy [10, 11]. Both aerobic and anaerobic exercises can be effective in the prevention and treatment of depressive symptoms [12, 13]. In a study by Thirlaway and Benton [14], participation in physical activities, rather than cardiovascular fitness, was associated with better mental health and mood.

A low level of physical exercise is known to be a risk factor for ischemic heart disease. Furthermore, depression is overrepresented among patients with ischemic heart disease. Its prevalence has been estimated to be as high as $30 \%$ [15]. Depression is also suggested to be an independent risk factor for ischemic cardiac disease [16] as well as a risk factor for increased mortality for cardiac patients [17]. Thus, cardiovascular morbidity might be a confounding factor while assessing the associations between depression and cardiovascular fitness.

Measurement of the maximal oxygen uptake $\left(\mathrm{VO}_{2 \mathrm{max}}\right)$ level is an objective method to assess cardiovascular fitness. To our knowledge, only one previous study has examined this association, reporting an inverse relationship between cardiovascular fitness and depression in a sample of 246 relatively young subjects [14]. Our aim was to examine whether there is an association between $\mathrm{VO}_{2 \max }$ and elevated depressive symptoms in a large population-based sample of middle-aged men. We excluded as far as possible the 
bias of cardiac diseases confounding the results by excluding cardiac patients and by adjusting the statistical models for confounding factors.

\section{Subjects and methods}

\section{Subjects}

The Kuopio Ischaemic Heart Disease Risk Factor (KIHD) study is an ongoing population-based study of risk factors for ischemic heart disease and other outcomes among middle-aged men in the Kuopio region of Eastern Finland [18]. A total of 2,678 participants aged $42-61$ years ( $82.9 \%$ of those eligible) were recruited. Those who reported having previously been diagnosed with a psychiatric disorder $(\mathrm{n}=156)$ were excluded. We also excluded men with a history of cancer $(\mathrm{n}=51)$ and/or cardiovascular disease other than high blood pressure $(n=1016)$, as these diseases might cause depression and a poor $\mathrm{VO}_{2 \max }$. Subjects with a $\mathrm{VO}_{2 \max }$ less than $15.8 \mathrm{ml} / \mathrm{kg}$ per min $(\mathrm{n}=73)$ were also excluded, because a very low $\mathrm{VO}_{2 \max }$ may be an indicator of an underlying but so far undiagnosed somatic disease. An exercise capacity of 5 metabolic equivalents (MET; equivalent to $15.8 \mathrm{ml} / \mathrm{kg}$ ) or less is related to a poor prognosis in terms of increased mortality in subjects younger than 65 years [19]. Data were incomplete for 25 participants, and thus complete data were available for 1,519 men. The study protocol was approved by the Research Ethics Committee of the University of Kuopio and Kuopio University Hospital.

\section{Assessment of cardiorespiratory fitness}

Maximal oxygen uptake $\left(\mathrm{VO}_{2 \max }, \mathrm{ml} / \mathrm{kg}\right.$ per min) was assessed between 1986 and 1989 by use of a maximal but symptom-limited exercise test on an electricallybraked cycle ergometer, as explained in detail elsewhere [20]. To obtain reliable information about exercise test variables and for safety reasons, the tests were supervised by an experienced physician assisted by an experienced nurse. The exercise tests were performed between 8:00 a.m. and 10:00 a.m. using a standardized testing protocol comprised of an increase in the workload by $20 \mathrm{~W} / \mathrm{min}$. Oxygen consumption was measured by using direct respiratory gas analyses. The $\mathrm{VO}_{2 \max }$ was defined as the highest value for or the plateau of oxygen uptake and was indexed by body weight.

\section{Assessment of depressive symptoms}

Depressive symptoms at the time of the measurements were assessed with the 18-item Human Population Laboratory Depression Scale (HPL depression scale) [21]. The scale consists of items dealing with mood disturbance, a negative self-concept, loss of energy, problems with eating and sleeping, trouble with concentration, and psychomotor agitation. The HPL score is generated by assigning one point for each true or false answer that is indicative of depression (range 0-18). In some items "often" or "never" responses, whichever were appropriate, were counted as one point. A cut-off score of five or more has earlier been used to define depression, and we therefore used the same cut-off point in this study. Those who scored five or more were considered to have elevated depressive symptoms $(\mathrm{n}=89,5.9 \%$ of participants). When the participants with somatic disease were included the number of participants with elevated depressive symptoms was 228 , representing $8.7 \%$ of the participants. The HPL depression scale has also previously been used to examine the association between depression and physical activity [1].

\section{Smoking and alcohol consumption}

The current number of cigarette, cigars, and pipefuls of tobacco smoked daily and the duration of regular smoking in years were recorded using a self-administered questionnaire. The number of years smoked was defined as the sum of the years of smoking, whether it had occurred continuously or during several periods. The lifelong exposure to smoking (pack years) was estimated as the product of years smoked and the number of tobacco products smoked daily at the time of the examination, or for ex-smokers at the time when they had last smoked. Alcohol consumption was assessed with a structured quantity-frequency method using the Nordic Alcohol Consumption Inventory on drinking behaviour over the previous 12 months and from the dietary record over 4 days.

\section{Other characteristics}

A variety of indicators of adulthood socioeconomic status (SES) were available, including current income, current and previous occupations, the highest level of education, the perception of financial security, and housing tenure. In addition, an index of material living conditions was created by summing the number of material possessions from a list of 12 (television, dishwasher, car, telephone, etc). The variable "adulthood socioeconomic status" (SES) was formed from these indicators [22]. Marital status was changed into a variable "living alone" (living in marriage or in common law marriage/living alone) and education into a two-class variable (graduated from high school/lower degree). The weight and height of the participants were measured and the body mass index (BMI) was calculated.

Physical activity was assessed using the 12-Month Physical Activity questionnaire [23-25]. The checklist included the most common physical activities of middle-aged men, for example walking, jogging, skiing, 
bicycling, swimming, and ball games. The subjects were asked to record the frequency, average duration, and intensity of each activity performed. A trained nurse checked and completed the questionnaire during an interview. The energy expenditure of physical activity was expressed as kcal per day.

Medical history, the use of medications and family history of diseases were assessed using self-administered questionnaires. Information about medical history and the use of medications were checked during a medical examination. Prevalent CHD was defined as having either a history of myocardial infarction, angina pectoris on effort or the use of nitroclycerin for chest pain once a week or more frequently. The prevalent CVD was defined as a history of CHD, hypertension, congestive heart failure, cardiomyopathy, arrhythmias, stroke or claudication.

\section{Statistics}

Differences in the characteristics between those participants with elevated depressive symptoms $(\mathrm{n}=89)$ and the rest of the cohort $(n=1424)$ were examined using the Student's $t$-test, Mann-Whitney $U$-test and $\chi^{2}$-test. Participants were grouped into quartiles according to their $\mathrm{VO}_{2 \max }$. Differences in characteristics between the quartiles of $\mathrm{VO}_{2 \max }$ were examined using ANOVA, the $\chi^{2}$-test and the Kruskal-Wallis test. The odds ratio of having elevated depressive symptoms was examined using a logistic regression model adjusted for age and examination years (Model 1) and further adjusted for alcohol consumption, marital status, adulthood SES and physical activity (Model 2). We also adjusted Model 1 for diastolic blood pressure, antihypertensive medication, diabetes and pack years of smoking to exclude the possibility of cardiovascular risk factors confounding our results (Model 3). Furthermore, we adjusted Model 1 for the items "less energy than other people" and "feeling too tired to do things" from the HLP depression scale to exclude the danger of circular reasoning (Model 4). We also used Spearman's $\rho$ to assess correlations between the severity of depressive symptoms and $\mathrm{VO}_{2 \max }$.

\section{Results}

The characteristics of the subjects with elevated depressive symptoms and the rest of the study population are presented in Table 1. Participants with elevated depressive symptoms were living alone more often and drank more alcohol than the other participants. They had also lower a $\mathrm{VO}_{2 \max }$ than other subjects. There was no difference in physical activity between participants with elevated depressive symptoms and the others. The characteristics of the study population according to the quartiles of maximal oxygen uptake are shown in Table $2 . \mathrm{VO}_{2 \max }$ was inversely associated with age, living alone, smoking, BMI, and waist to hip ratio.

The proportion of participants with elevated depressive symptoms was $9.0 \%$ in the lowest quartile $(15.8-28.1 \mathrm{ml} / \mathrm{kg} / \mathrm{min})$ of $\mathrm{VO}_{2 \max }$ and $2.8 \%$ in the highest quartile (36.2-65.4 ml $/ \mathrm{kg} / \mathrm{min}$; Table 2). A high $\mathrm{VO}_{2 \max }$ was associated with higher physical activity (Table 2.). Participants in the lowest quartile of $\mathrm{VO}_{2 \max }$ had a 3.4-fold higher risk of having elevated depressive symptoms than the men in highest quartile (Table 3, Model 1). After further adjustment for smoking habits, alcohol consumption, marital status, adulthood SES, and physical activity, the risk remained statistically significant (Table 3 , Model 2).

Table 1. Characteristics ${ }^{1}$ of the study population according to the presence (HPL $\left.\geq 5 ; \mathrm{n}=89\right)^{2}$ or absence (HPL $<5$; $\mathrm{n}=1424)$ of current elevated depressive symptoms

\begin{tabular}{llll}
\hline & $(\mathrm{HPL} \geq 5)$ & $(\mathrm{HPL}<5)$ & $p$ Value \\
\hline $\mathrm{N}$ & & & $0.567^{3}$ \\
Age (years) & $52.4(5.3)$ & $52.0(5.5)$ & $0.005^{4}$ \\
Alcohol/week (g) & $95.3(118.3)$ & $72.4(114.6)$ & $0.377^{3}$ \\
Body mass index (BMI) kg/m $26.6(3.4)$ & $0.383^{3}$ \\
Waist to hip ratio & $26.9(4.2)$ & $0.94(0.06)$ & $0.430^{3}$ \\
Energy (MJ/day) & $0.95(0.06)$ & $10.11(2.65)$ & $0.727^{3}$ \\
Daily intake of fat (g) & $9.89(2.64)$ & $103.4(34.1)$ & $0.005^{5}$ \\
Marital status: living alone (\%) & $102.1(33.7)$ & 12.2 & $0.697^{5}$ \\
Smoking (\%) & 22.5 & 30.6 & $0.001^{3}$ \\
Maximal oxygen uptake (ml/kg per min) & 33.6 & $32.8(7.2)$ & $0.106^{4}$ \\
Physical activity (kcal/d) & $30.3(5.8)$ & $137.6(154.6)$ & \\
\hline
\end{tabular}

Significance of the difference between groups assessed by:.

1 Values are means (SD) or \%.

${ }^{2}$ HPL is Human Population Laboratory Depression Scale.

3 Student's $t$-test.

4 Mann-Whitney $U$-test.

$\chi^{2}$-test. 
Table 2. Characteristics ${ }^{1}$ of the study population $(n=1513)$ divided into quartiles according to maximal oxygen uptake

\begin{tabular}{lllllc}
\hline & First quartile & Second quartile & Third quartile & Fourth quartile & $\begin{array}{c}p \text { Value for } \\
\text { difference }\end{array}$ \\
\hline Range (ml/kg per min) & & & & & \\
Age (years) & $15.8-28.1$ & $28.1-31.3$ & $31.3-36.2$ & $36.2-65.4$ & $<.3$ \\
Alcohol/week (g) & $54.2(4.6)$ & $52.9(5.3)$ & $51.7(5.3)$ & $49.5(5.5)$ & $<0.001^{2}$ \\
Body mass index (BMI) kg/m & $84.7(127.1)$ & $94.1(158.1)$ & $66.4(88.1)$ & $55.6(82.3)$ & $0.088^{3}$ \\
Waist to hip ratio & $28.3(3.8)$ & $26.9(3.6)$ & $26.3(2.8)$ & $25.0(2.4)$ & $<0.001^{2}$ \\
Living alone (\%) & $0.97(0.05)$ & $0.95(0.05)$ & $0.94(0.5)$ & $0.92(0.6)$ & $<0.001^{2}$ \\
Smoking (\%) & 16.2 & 13.6 & 11.6 & 10.3 & $0.010^{4}$ \\
Physical activity (kcal/d) & 38.3 & 31.9 & 31.6 & 21.9 & $<0.001^{4}$ \\
Number of participants & $110.6(130.9)$ & $126.9(149.5)$ & $116.4(140.7)$ & $189.6(186.5)$ & $<0.0001^{3}$ \\
with elevated depressive symptoms (\%) & $33(9.0)$ & $26(6.8)$ & $19(5.0)$ & $11(2.8)$ & $<0.001^{4}$ \\
\hline
\end{tabular}

1 Values are means (SD) or \%.

2 ANOVA.

${ }^{3}$ Kruskal-Wallis test.

${ }^{4} \chi^{2}$-test, $p$ for linear trend.

We further adjusted Model 1 for diabetes, diastolic blood pressure, hypertensive medication and pack years of smoking (Model 3) and for the items "less energy than other people" and "feeling too tired to do things" from the HLP depression scale (Model 4). Those in the lowest quartile still had a significantly higher risk of having elevated depressive symptoms than those in the highest quartile (Model 3: OR: 2.98; 95\% CI: 1.40-6.34; $p<0.005$; Model 4: OR: 2.63; 95\% CI: $1.21-5.69 ; p<0.015)$.

To study the effect of diagnosed somatic illness we repeated our analysis without any exclusions $(n=2626)$. However, this did not result in a significant change in the findings (Model 1: OR: 3.64; 95\% CI: $2.38-5.55 ; p<0.0001$; Model 2: OR: 3.22; $95 \%$ CI: 2.09-4.95; $p<0.0001)$.

Furthermore, there was an inverse correlation between the HPL depression score and $\mathrm{VO}_{2 \max }$ $(\rho=-0.160, p<0.0001)$. After inclusion of the participants with somatic disease the result remained significant and the correlation was even stronger $(\mathrm{n}=2626 ; \rho=-0.223, \quad p<0.0001)$. We also examined the correlation between the HPL depression scale and $\mathrm{VO}_{2 \max }$ after the exclusion of items "less energy than other people" and "feeling too tired to do things". This had little effect on the correlations $(\rho=-0.155, \quad p<0.0001 ; \mathrm{n}=2626, \quad \rho=-0.217$, $p<0.0001$, respectively).

\section{Discussion}

Maximal oxygen uptake was inversely related to elevated depressive symptoms in a population-based sample of middle-aged men. In this study, participants with a low oxygen uptake of less than $28.1 \mathrm{ml} /$ $\mathrm{kg} / \mathrm{min}$ had 3.4-fold higher risk of being depressed compared with those with a $\mathrm{VO}_{2 \max }$ exceeding $36.2 \mathrm{ml} / \mathrm{kg} / \mathrm{min}$, even after adjustment for alcohol consumption, adulthood SES, marital status, education, BMI, and physical activity. Furthermore, there was a correlation between the severity of the depression scores and cardiovascular fitness.

Our results somewhat contradict the finding of Thirlaway and Benton [14] that physical activity rather than $\mathrm{VO}_{2 \max }$ was associated with depression. However, in their study an increased $\mathrm{VO}_{2 \max }$ was associated with better mood in a physically inactive sub-group of study subjects. Thirlaway and Benton [14] suggested that those who were fit but physically inactive had previously been physically active, but the subjects had only been asked about their inactivity

Table 3. Odds ratios of elevated depressive symptoms according to the quartile for maximal oxygen uptake

\begin{tabular}{|c|c|c|c|c|}
\hline & $\begin{array}{l}\text { First quartile Model } 1^{1} \\
\text { [(Model 22] OR }(95 \% \text { CI })\end{array}$ & $\begin{array}{l}\text { Second quartile Model } 1^{1} \\
{\left[\text { Model } 2^{2}\right] \text { OR }(95 \% \text { CI) }}\end{array}$ & $\begin{array}{l}\text { Third quartile Model } 1^{1} \\
{[\text { Model 2²] OR (95\% CI) }}\end{array}$ & $\begin{array}{l}\text { Fourth } \\
\text { quartile OR }\end{array}$ \\
\hline $\begin{array}{l}\text { Risk of elevated } \\
\text { depressive } \\
\text { symptoms }\end{array}$ & $\begin{array}{l}3.42(1.65-7.09, p<0.001) \\
{[3.38(1.60-7.14), p=0.001]}\end{array}$ & $\begin{array}{l}2.45(1.16-5.18, p=0.019) \\
{[2.37(1.01-5.10], p=0.027]}\end{array}$ & $\begin{array}{l}1.80(0.84-3.87, p=0.130) \\
{[1.81(0.82-3.95), p=0.113]}\end{array}$ & 1.0 (ref.) \\
\hline
\end{tabular}

\footnotetext{
1 Logistic regression adjusted for age and examination years.

2 Logistic regression adjusted for examination years, smoking habits, alcohol consumption, marital status, adulthood socioeconomic status, and physical activity.

3 -values are for the difference compared with the fourth quartile.
} 
over the preceding 2 weeks. Another possible explanation for their findings could be that current physical activity could compensate for the effect of poor fitness on mood.

It is possible that $\mathrm{VO}_{2 \max }$ reflects the cumulative effect of life-long physical activity. However, in our study self-reported physical activity did not correlate with depression. This could mostly be explained by the genetic contribution to variation in fitness. It has been reported that the genetic contribution to aerobic power might be approximately $25-40 \%$ [26-28]. In our study, physical activity associated with a higher $\mathrm{VO}_{2 \max }$, which indicates the impact of the exercise on the $\mathrm{VO}_{2 \max }$. However, although for most individuals an increase in physical exercise produces an increase in the $\mathrm{VO}_{2 \max }$, the increase for a standard exercise dose varies widely and is under genetic control [28]. Self-reported questionnaires may only reliably reflect short-term exercise habits, whereas the $\mathrm{VO}_{2 \max }$ is the result of long-term habits and the genetic background.

This was a large population-based study, which can be considered strength. We were able to exclude several somatic disorders that might be associated with both depression and poor cardio-respiratory fitness. We also adjusted the models for various lifestyle factors, including physical activity.

\section{Limitations}

We did not use structured diagnostic interviews to diagnose depression, which may be considered a limitation. Some of the study participants who were classified as having an elevated level of depressive symptoms might not have qualified for the diagnosis of depressive disorder. However, the HPL depression scale has especially been developed for screening depression in general population samples $[29,30]$ and is highly correlated with the Beck Depression Inventory score [21, 31].

In a cross-sectional study we cannot conclusively determine whether poor cardiovascular fitness is a cause or a consequence of elevated depressive symptoms. If the participants having an elevated level of depressive symptoms had been depressed for a long time this may have affected their lifestyle and physical activity. To avoid this bias we excluded those subjects who had previously been diagnosed with clinical depression. However, it is possible that current elevated depressive symptoms might have some effect on the result of the bicycle ergometer test due to poor motivation. A healthy lifestyle and general health may also be commonly connected with both mental health and fitness, and thus the maximal oxygen uptake could be a factor that has no direct causal effect on mood. To avoid this possible bias we adjusted the models for several life-style and psychological variables known to associate with depression. Finally, despite of all our adjustments we cannot completely rule out the possibility of sub-clinical conditions confounding our results. Also, our sample included only men, but previous studies have detected an association between physical activity and depression in both men and women [4, 14].

\section{Conclusion}

Poor cardiovascular fitness was associated with elevated depressive symptoms in middle-aged men. Further prospective intervention studies are needed to conclusively verify whether this association is causal. Further studies are also needed to assess the mechanisms underlying this association. Maximal oxygen uptake has a strong genetic contribution, but it can be increased by physical exercise.

\section{Human participant protection}

The study protocol was approved by the Research Ethics Committee of the University of Kuopio.

\section{References}

1. Camacho TC, Roberts RE, Lazarus NB, Kaplan GA, Cohen RD. Physical activity and depression: evidence from the Alameda County Study. Am J Epidemiol 1991; 134: 220-231.

2. Paffenbarger R Jr, Lee I, Leung R. Physical activity and personal characteristics associated with depression and suicide in American college men. Acta Psychiatr Scand 1994; 377(suppl): 16-22.

3. Hassmén P, Koivula N, Uutela A. Physical exercise and psychological well-being: A population study in Finland. Prev Med 2000; 30: 17-25.

4. Strawbridge W, Deleger S, Roberts R, Kaplan G. Physical activity reduces the risk of subsequent depression for older adults. Am J Epidemiol 2002; 156: 328-334.

5. Stephens T. Physical activity and mental health in the United States and Canada: Evidence from four population surveys. Prev Med 1988; 17: 35-47.

6. Steptoe A, Edwards S, Moses J, Mathews A. The effects of exercise training on the mood and perceived coping ability in anxious adults from the general population. J Psychosom Res 1989; 33: 537-547.

7. Lampinen P, Heikkinen R-L, Ruoppila R. Changes in Intensity of Physical Exercise as Predictors of Depressive Symptoms Among Older Adults: An eight-year follow-up. Prev Med 2000; 30: 371-380.

8. Martinsen E. Physical activity and depression: Clinical experience. Acta Psychiatr Scand 1994; 377(suppl): 23 27.

9. Veale D, Le Fevre K, Pantelis C, de Souza V, Mann A, Sargeant A. Aerobic exercise in the adjunctive treatment of depression: A randomized controlled trial. J R Soc Med 1992; 85: 541-544.

10. Blomenthal J, Babyak M, Moore K, et al. Effects of exercise training on older adults with major depression. Arch Intern Med 1999; 159: 2349-2356. 
11. Babyak M, Blumenthal J, Herman S, et al. Exercise treatment for major depression: Maintenance of therapeutic benefit at 10 months. Psychosom Med 2000; 62: 633-638.

12. Doyne E, Ossip-Klein D, Bowman E, Osborn K, McDougall-Wilson I, Neimeyer R. Running versus weight lifting in the treatment of depression. J Consult Clin Psychol 1987; 55: 748-754.

13. Martinsen E, Hoffart A, Solberg O. Comparing aerobic with nonaerobic forms of exercise in the treatment of clinical depression: a randomized trial. Compr Psychiatry 1989 ; 30: 324-331.

14. Thirlaway K, Benton D. Participation in physical activity and cardiovascular fitness have different effects on mental health and mood. J Psychosom Res 1992; 36: $657-665$.

15. Lespérance F, Frasure-Smith N. Depression in patients with cardiac disease: A practical review. J Psychosom Res 2000; 48: 379-391.

16. Hemingway $\mathrm{H}$, Marmot M. Psychosocial factors in the aetiology and prognosis of coronary heart disease: Systematic review of prospective cohort studies. $\mathrm{Br}$ Med J 1999; 318: 1460-1467.

17. Kauffmann MW, Fitzgibbons JP, Sussman EJ, et al. Relation between myocardial infarction, depression, hostility, and death. Am Heart J 1999; 138: 549-554.

18. Salonen JT. Is there a continuing need for longitudinal epidemiological research? The Kuopio Ischaemic Heart Disease Risk Factor Study. Ann Clin Res 1988; 20: 46-50.

19. Fletcher GF, Balady G, Froelicher VF, Hartley H, Haskell WL, Pollock ML. A statement for healthcare professionals from the American Heart Association: Exercise standards. Circulation 1992; 86: 340-344.

20. Lakka TA, Venäläinen JM, Rauramaa R, Salonen R, Tuomilehto J, Salonen JT. Relation of leisure-time physical activity and cardiorespiratory fitness to the risk of acute myocardial infarct. N Engl J Med 1994; 330: 1549-1554.

21. Kaplan GA, Roberts RE, Camacho TC, Coyne JC. Psychosocial predictors of depression: Prospective evidence from the Human Population Laboratory Studies. Am J Epidemiol 1987; 125: 206-220.
22. Wilson TW, Kaplan GA, Kauhanen J, et al. The association between plasma fibrinogen concentration and five socioeconomic indices in the Kuopio Ischeamic Heart Disease Risk Factor Study. Am J Epidemiol 1993; 137: 292-300.

23. Salonen JT, Lakka TA. Assessment of physical activity in population studies - validity and consistency of the methods in the Kuopio Ischeamic Heart Study. Scand J Sport Sci 1987; 9: 89-95.

24. Ainsworth BE, Haskell WL, Leon AS, et al. Compendium of physical activities: Classification of energy costs of human physical activities. Med Sci Sports Exerc 1993; 25: 71-80.

25. Lakka TA, Salonen JT. Intra-Person variability of various physical activity assessments in the Kuopio Ischeamic Heart Risk Factor Study. Int J Epidemiol 1992; 21: 467-472.

26. Bouchard C, Rankinen T, Chagnon YC, et al. Genomic scan for maximal oxygen uptake and its response to training in the HERITAGE Family Study. J Appl Physiol 2000; 88: 551-559.

27. Perusse L, Rice T, Province MA, et al. Familiar aggregation of amount and distribution of subcutaneous fat and their responses to exercise training in the HERITAGE family study. Obes Res 2000; 8: 140-150.

28. Bouchard C, Rankinen T. Individual differences in response to regular physical activity. Med Sci Sports Exerc 2001; 33: 446-451.

29. Roberts RE. Prevalence of depressive symptoms among Mexican Americans. J Nerv Ment Dis 1981; 169: 213-219.

30. Roberts RE, O'Keefe SJ. Sex differences in depression re-examined. J Health Soc Behav 1981; 22: 394-400.

31. Beck AT, Ward CH, Mendelson M. An inventory for measuring depression. Arch Gen psychiatry 1961; 4: $561-571$.

Address for correspondence: T. Tolmunen, Department of Psychiatry, Kuopio University Hospital, P.O. Box 1777, FIN-70211, Kuopio, Finland

Phone: + 358-40-8277 523

E-mail: tommi.tolmunen@kuh.fi 